\title{
Explicit formulas for biharmonic submanifolds in non-Euclidean 3-spheres
}

\author{
D. FETCU and C. ONICIUC* \\ Dedicated to Professor Vasile Oproiu on his 65-th birthday
}

\begin{abstract}
We obtain the parametric equations of all biharmonic Legendre curves and Hopf cylinders in the 3-dimensional unit sphere endowed with the modified Sasakian structure defined by Tanno.

2000 MSC: 53C42, 53B25.

Key words: Biharmonic submanifolds, Sasakian space forms, Legendre curves, Hopf cylinders.
\end{abstract}

\section{Introduction}

Biharmonic maps $\phi:(M, g) \rightarrow(N, h)$ between Riemannian manifolds are the critical points of the bienergy functional $E_{2}(\phi)=\frac{1}{2} \int_{M}|\tau(\phi)|^{2} \nu_{g}$ and represent a natural generalization of the well-known harmonic maps, the critical points of the energy functional $E(\phi)=\frac{1}{2} \int_{M}|d \phi|^{2} \nu_{g}$. The bienergy functional has been suggested since 1964 by Eells and Sampson in their famous paper [13]. The bienergy functional can be viewed as the analogue for maps of the Willmore functional for Riemannian immersions. In a different setting, Chen defined the biharmonic submanifolds in an Euclidean space. If we apply the characterization formula of biharmonic maps to Riemannian immersions into Euclidean spaces, we recover Chen's notion of biharmonic submanifold.

The Euler-Lagrange equation for the energy functional is $\tau(\phi)=0$, where $\tau(\phi)=\operatorname{trace} \nabla d \phi$ is the tension field, and the Euler-Lagrange equation for the bienergy functional was derived by Jiang in [15]:

$$
\begin{aligned}
\tau_{2}(\phi) & =-\Delta \tau(\phi)-\operatorname{trace} R^{N}(d \phi, \tau(\phi)) \tau(\phi)= \\
& =0 .
\end{aligned}
$$

${ }^{*}$ The authors were partially supported by the Grant CEEX, ET, 5871/2006, Romania. 
Since any harmonic map is biharmonic, we are interested in non-harmonic biharmonic maps, which are called proper-biharmonic.

There are several classification results of proper-biharmonic submanifolds in 3dimensional spaces. The proper-biharmonic submanifolds in $\mathbb{R}^{3}, \mathbb{S}^{3}$ and $N^{3}(-1)$, the space of constant negative sectional curvature -1 , were completely classified in [11, [5] and [6, respectively. Then, the next step was to classify properbiharmonic submanifolds in a 3-dimensional space of non-constant sectional curvature. In [8], [7] and [12] the authors classified the proper-biharmonic curves in the Heisenberg group and Cartan-Vranceanu 3-dimensional spaces, while, in [14], Inoguchi classified the proper-biharmonic Legendre curves and Hopf cylinders in a 3-dimensional Sasakian space form $M^{3}(c)$.

For a general account of biharmonic maps see [16] and The bibliography of biharmonic maps [18].

The goal of this paper is to obtain explicitly the parametric equations of proper-biharmonic Legendre curves and Hopf cylinders in $\mathbb{S}^{3}$ with the modified Sasakian structure defined by Tanno, by using the results in [14]. The techniques used in our paper are introduced in [1] and [2].

Conventions. We work in the $C^{\infty}$ category, that means manifolds, metrics, connections and maps are smooth. The Lie algebra of the vector fields on $M$ is denoted by $C(T M)$.

\section{Preliminaries}

\subsection{Contact manifolds}

A contact metric structure on a manifold $M^{2 n+1}$ is given by $(\varphi, \xi, \eta, g)$, where $\varphi$ is a tensor field of type $(1,1)$ on $M, \xi$ is a vector field on $M, \eta$ is an 1-form on $M$ and $g$ is a Riemannian metric, such that

$$
\left\{\begin{array}{l}
\varphi^{2}=-I+\eta \otimes \xi, \quad \eta(\xi)=1, \\
g(\varphi X, \varphi Y)=g(X, Y)-\eta(X) \eta(Y), \quad \forall X, Y \in C(T M) . \\
g(X, \varphi Y)=d \eta(X, Y), \quad \forall X, Y \in C(T M)
\end{array}\right.
$$

A contact metric structure $(\varphi, \xi, \eta, g)$ is Sasakian if it is normal, that is

$$
N_{\varphi}+2 d \eta \otimes \xi=0
$$

where

$$
N_{\varphi}(X, Y)=[\varphi X, \varphi Y]-\varphi[\varphi X, Y]-\varphi[X, \varphi Y]+\varphi^{2}[X, Y], \quad \forall X, Y \in C(T M),
$$


is the Nijenhuis tensor field of $\varphi$, or, equivalently, if

$$
\left(\nabla_{X} \varphi\right)(Y)=g(X, Y) \xi-\eta(Y) X, \quad \forall X, Y \in C(T M) .
$$

Let us consider a Sasakian manifold $(M, \varphi, \xi, \eta, g)$. The sectional curvature of a 2-plane generated by $X$ and $\varphi X$, where $X$ is an unit vector orthogonal to $\xi$, is called $\varphi$-sectional curvature determined by $X$. A Sasakian manifold with constant $\varphi$-sectional curvature $c$ is called a Sasakian space form and it is denoted by $M(c)$.

The curvature tensor field of a Sasakian space form $M(c)$ is given by

$$
\begin{aligned}
R(X, Y) Z= & \frac{c+3}{4}\{g(Z, Y) X-g(Z, X) Y\}+\frac{c-1}{4}\{\eta(Z) \eta(X) Y- \\
& -\eta(Z) \eta(Y) X+g(Z, X) \eta(Y) \xi-g(Z, Y) \eta(X) \xi+ \\
& +g(Z, \varphi Y) \varphi X-g(Z, \varphi X) \varphi Y+2 g(X, \varphi Y) \varphi Z\} .
\end{aligned}
$$

A contact metric manifold $(M, \varphi, \xi, \eta, g)$ is called regular if for any point $p \in M$ there exists a cubic neighborhood of $p$ such that any integral curve of $\xi$ passes through the neighborhood at most once, and strictly regular if all integral curves are homeomorphic to each other.

Let $(M, \varphi, \xi, \eta, g)$ be a regular contact metric manifold. Then the orbit space $\bar{M}$ can be naturally organized as a manifold and, moreover, if $M$ is compact then $M$ is a principal circle bundle over $\bar{M}$ (the Boothby-Wang Theorem). In this case the fibration $\pi: M \rightarrow \bar{M}$ is called the Boothby-Wang fibration. A very known example of a Boothby-Wang fibration is the Hopf fibration $\pi: \mathbb{S}^{2 n+1} \rightarrow \mathbb{C} P^{n}$.

We recall the following result obtained by Ogiue

Theorem 2.1 ([17]) Let $(M, \varphi, \xi, \eta, g)$ be a strictly regular Sasakian manifold. Then $\bar{M}$ can be organized as a Kähler manifold. Moreover, if $(M, \varphi, \xi, \eta, g)$ is a Sasakian space form $M(c)$, then $\bar{M}$ has constant sectional holomorphic curvature $c+3$.

\subsection{General results}

In [5] all biharmonic curves and surfaces in the 3-dimensional Euclidean unit sphere $\left(\mathbb{S}^{3}, g_{0}\right)$, where $g_{0}$ is the usual metric, were classified:

Theorem 2.2 ([5]) Let $M^{m}$ be a proper-biharmonic submanifold of $\mathbb{S}^{3}$. We have

a) if $m=1$, then $M$ is either the circle of radius $\frac{1}{\sqrt{2}}$ or a geodesic of the torus $\mathbb{S}^{1}\left(\frac{1}{\sqrt{2}}\right) \times \mathbb{S}^{1}\left(\frac{1}{\sqrt{2}}\right) \subset \mathbb{S}^{3}$ with the slope different from \pm 1 ;

b) if $m=2$, then $M$ is the hypersphere $\mathbb{S}^{2}\left(\frac{1}{\sqrt{2}}\right) \subset \mathbb{S}^{3}$. 
We can think $\left(\mathbb{S}^{3}, g_{0}\right)$ as a Sasakian space form with constant $\varphi_{0}$-sectional curvature 1. We know that in the geometry of Sasakian manifolds an important role is played by the integral submanifolds and Hopf cylinders. A natural question is whether biharmonic Legendre curves and Hopf cylinders in $\left(\mathbb{S}^{3}, \varphi_{0}, \xi_{0}, \eta_{0}, g_{0}\right)$ exist. Since the torsion of a Legendre curve in $\left(\mathbb{S}^{3}, \varphi_{0}, \xi_{0}, \eta_{0}, g_{0}\right)$ is 1 and a Hopf cylinder is flat, it follows that in $\left(\mathbb{S}^{3}, \varphi_{0}, \xi_{0}, \eta_{0}, g_{0}\right)$ do not exist proper-biharmonic Legendre curves and proper-biharmonic Hopf cylinders.

The next step is the study of the existence of biharmonic Legendre curves and of biharmonic Hopf cylinders in Sasakian space forms with constant $\varphi$-sectional curvature $c \neq 1$. Inoguchi gave the following classification:

Theorem 2.3 ([14]) Let $M^{3}(c)$ be a Sasakian space form of constant $\varphi$-sectional curvature $c$ and let $\gamma: I \rightarrow M$ be a Legendre curve parametrized by arc length. We have

a) if $c \leqslant 1$, then $\gamma$ is biharmonic if and only if it is geodesic;

b) if $c>1$, then $\gamma$ is proper-biharmonic if and only if it is a helix with the curvature $\bar{\kappa}^{2}=c-1$.

Theorem 2.4 ([14]) Let $M^{3}(c)$ be a Sasakian space form of constant $\varphi$-sectional curvature $c$ and $S_{\bar{\gamma}}$ a Hopf cylinder, where $\bar{\gamma}$ is a curve in the orbit space of $M^{3}(c)$, parametrized by arc length. We have

a) if $c \leqslant 1$, then $S_{\bar{\gamma}}$ is biharmonic if and only if it is minimal;

b) if $c>1$, then $S_{\bar{\gamma}}$ is proper-biharmonic if and only if the curvature $\bar{\kappa}$ of $\bar{\gamma}$ is constant $\bar{\kappa}^{2}=c-1$.

Let $M^{3}(c)$ be a Sasakian space form with constant $\varphi$-sectional curvature $c>1$, and let $\bar{\gamma}: I \rightarrow \bar{M}$ be a curve parametrized by arc length. We denote $f_{1}=\bar{\gamma}^{\prime}$ and $f_{2}=\bar{J} f_{1}$ the Frenet frame field along $\bar{\gamma}$, where $\bar{J}$ is the almost complex structure on $\bar{M}$. Consider $S_{\bar{\gamma}}=\pi^{-1}(\bar{\gamma})$ the Hopf cylinder corresponding to $\bar{\gamma}$ and assume that it is biharmonic, that is $\bar{k}^{2}=c-1$. Since $\left[\xi, f_{1}^{H}\right]=0, g(\xi, \xi)=1$, $g\left(f_{1}^{H}, f_{1}^{H}\right)=1$, we can choose a local chart $x=x(u, v)$ such that $\xi=x_{v}$ and $f_{1}^{H}=x_{u}$. The parametric curves $u \rightarrow x\left(u, v_{0}\right)$ are proper-biharmonic Legendre curves parametrized by arc length and geodesics in $S_{\bar{\gamma}}$. Moreover, $u \rightarrow x\left(u, v_{0}\right)$ are the only geodesics of $S_{\bar{\gamma}}$ which are proper-biharmonic in $M^{3}(c)$.

Proposition 2.5 Let $M^{3}(c)$ be a Sasakian space form with constant $\varphi$-sectional curvature $c>1$ and $S_{\bar{\gamma}}$ a biharmonic Hopf cylinder. Let $\gamma: I \rightarrow S_{\bar{\gamma}}$ be a geodesic parametrized by arc length and $\mathbf{i}: S_{\bar{\gamma}} \rightarrow M^{3}(c)$ the canonical inclusion. Then $\mathbf{i} \circ \gamma: I \rightarrow M^{3}(c)$ is proper-biharmonic if and only if $\gamma^{\prime}= \pm f_{1}^{H}$. 
Proof. Let $\gamma: I \rightarrow S_{\bar{\gamma}}$ be a geodesic parametrized by arc length. Then $\gamma^{\prime}=c_{1} \xi+c_{2} f_{1}^{H}$, where $c_{1}$ and $c_{2}$ are real constants such that $c_{1}^{2}+c_{2}^{2}=1$ and $f_{1}^{H}$ is the horizontal lift of $f_{1}$. After a straightforward computation one obtains

$$
\tau(\mathbf{i} \circ \gamma)=c_{2}\left(c_{2} \bar{\kappa}-2 c_{1}\right) f_{2}^{H} \text { and } \tau_{2}(\mathbf{i} \circ \gamma)=2 c_{1} c_{2}^{2}\left(c_{2} \bar{\kappa}-2 c_{1}\right) \bar{\kappa} f_{2}^{H},
$$

where $\bar{\kappa}^{2}=c-1$. Hence $\tau(\mathbf{i} \circ \gamma) \neq 0$ and $\tau_{2}(\mathbf{i} \circ \gamma)=0$ if and only if $c_{1}=0$, that is $\gamma^{\prime}= \pm f_{1}^{H}$.

It can be easily proved that, if $\gamma: I \rightarrow M^{3}(c)$ is a biharmonic Legendre curve, then it is a geodesic in the biharmonic Hopf cylinder $x(s, t)=\phi_{\gamma(s)}(t)$, where $\left\{\phi_{t}\right\}$ is the uniparametric group of $\xi$. From now on we will use the parameters $(u, v)$ instead of $(s, t)$.

In the following we will choose the 3 -sphere $\mathbb{S}^{3}$ with modified Sasakian structure as a model for the complete, simply connected Sasakian space form with constant $\varphi$-sectional curvature $c>1$, and we will find the explicit equations of biharmonic Legendre curves and of biharmonic Hopf cylinders, viewed as submanifolds of $\mathbb{R}^{4}$. These equations are given by Theorem 3.1 and Theorem 4.1 , respectively.

\section{Proper-biharmonic Legendre curves}

Let $\mathbb{S}^{3}=\left\{z \in \mathbb{C}^{2}:\|z\|=1\right\}$ be the unit 3-dimensional sphere endowed with its standard metric field $g_{0}$. Consider the following structure tensor fields on $\mathbb{S}^{3}$ : $\xi_{0}=-J z$ for each $z \in \mathbb{S}^{3}$, where $J$ is the usual almost complex structure on $\mathbb{C}^{2}$ defined by $J z=\left(-y_{1},-y_{2}, x_{1}, x_{2}\right)$ for $z=\left(x_{1}, x_{2}, y_{1}, y_{2}\right)$, and $\varphi_{0}=s \circ J$, where $s: T_{z} \mathbb{C}^{2} \rightarrow T_{z} \mathbb{S}^{3}$ denotes the orthogonal projection. Endowed with these tensors, $\mathbb{S}^{3}$ becomes a Sasakian space form with $\varphi_{0}$-sectional curvature 1 (see [4]). Now we consider the deformed structure

$$
\eta=a \eta_{0}, \quad \xi=\frac{1}{a} \xi_{0}, \quad \varphi=\varphi_{0}, \quad g=a g_{0}+a(a-1) \eta_{0} \otimes \eta_{0},
$$

where $a$ is a positive constant. Such a deformation is called a $\mathcal{D}$-homothetic deformation, since the two metrics restricted to the contact subbundle $\mathcal{D}$ are homothetic, and it was introduced in [19]. The deformed structure $(\varphi, \xi, \eta, g)$ is still a Sasakian structure and $\left(\mathbb{S}^{3}, \varphi, \xi, \eta, g\right)$ is a Sasakian space form with constant $\varphi$-sectional curvature $c=\frac{4}{a}-3$ (see [2]). From now on we assume that $a \in(0,1)$, that is $c>1$. 
Theorem 3.1 Let $\gamma: I \rightarrow\left(\mathbb{S}^{3}, \varphi, \xi, \eta, g\right)$ be a Legendre curve parametrized by arc length. Then it is proper-biharmonic if and only if, as a curve in $\mathbb{R}^{4}$

$$
\begin{aligned}
\gamma(s)= & \sqrt{\frac{B}{A+B}} \cos (A s) e_{1}-\sqrt{\frac{B}{A+B}} \sin (A s) J e_{1}+ \\
& +\sqrt{\frac{A}{A+B}} \cos (B s) e_{3}+\sqrt{\frac{A}{A+B}} \sin (B s) J e_{3},
\end{aligned}
$$

where $\left\{e_{1}, e_{3}\right\}$ is an orthonormal system of constant vectors in the Euclidian space $\left(\mathbb{R}^{4},\langle\rangle,\right)$, with $e_{3}$ orthogonal to $J e_{1}$, and

$$
\left\{\begin{array}{l}
A=\sqrt{\frac{3-2 a-2 \sqrt{(a-1)(a-2)}}{a}} \\
B=\sqrt{\frac{3-2 a+2 \sqrt{(a-1)(a-2)}}{a}}
\end{array} .\right.
$$

Proof. Let us denote by $\nabla, \dot{\nabla}$ and $\widetilde{\nabla}$ the Levi-Civita connections on $\left(\mathbb{S}^{3}, g\right)$, $\left(\mathbb{S}^{3}, g_{0}\right)$ and $\left(\mathbb{R}^{4},\langle\rangle,\right)$, respectively.

Let $\gamma: I \rightarrow\left(\mathbb{S}^{3}, g\right)$ be a proper-biharmonic Legendre curve parametrized by arc length and let $T=\gamma^{\prime}$ be the unit tangent vector field along the curve. Using Theorem 2.3 we have

$$
\bar{g}(T, T)=1, \quad g(T, \xi)=0, \quad \nabla_{T} T=\kappa \varphi T, \quad \text { and } \quad \kappa^{2}=c-1 .
$$

In order to find the explicit parametrization of $\gamma$ as a curve in $\mathbb{R}^{4}$ we shall characterize $\gamma$ as the solution of a certain fourth order ODE with constant coefficients. For this purpose the expressions of $\widetilde{\nabla}_{T} T, \widetilde{\nabla}_{T} \varphi T$ and $\widetilde{\nabla}_{T} \xi$ are needed.

First, we observe that $g\left(\nabla_{X} X, Z\right)=a g_{0}\left(\dot{\nabla}_{X} X, Z\right)$, for any $Z \in C\left(T \mathbb{S}^{3}\right)$ and for any $X \in C\left(T \mathbb{S}^{3}\right)$ orthogonal to $\xi$. Then, using the properties of the Sasakian structures $(\varphi, \xi, \eta, g)$ and $\left(\varphi_{0}, \xi_{0}, \eta_{0}, g_{0}\right)$, we get

$$
\dot{\nabla}_{T} T=\kappa \varphi T, \quad \dot{\nabla}_{T} \varphi T=-\kappa T+\xi, \quad \dot{\nabla}_{T} \xi=-\frac{1}{a} \varphi T .
$$

Further, using the Gauss equation of $\left(\mathbb{S}^{3}, g_{0}\right)$ in $\left(\mathbb{R}^{4},\langle\rangle,\right)$, we obtain

$$
\left\{\begin{array}{c}
\widetilde{\nabla}_{T} T=\dot{\nabla}_{T} T-\langle T, T\rangle \gamma=\kappa \varphi T-\frac{1}{a} \gamma \\
\widetilde{\nabla}_{T} \varphi T=-\kappa T+\xi, \quad \widetilde{\nabla}_{T} \xi=-\frac{1}{a} \varphi T
\end{array} .\right.
$$

Now it follows

$$
\widetilde{\nabla}_{T} \widetilde{\nabla}_{T} T=\kappa \widetilde{\nabla}_{T} \varphi T-\frac{1}{a} T=-\left(\frac{1}{a}+\kappa^{2}\right) T+\kappa \xi
$$


and

$$
\widetilde{\nabla}_{T} \widetilde{\nabla}_{T} \widetilde{\nabla}_{T} T=-\left(\frac{2}{a}+\kappa^{2}\right)\left(\widetilde{\nabla}_{T} T+\frac{1}{a} \gamma\right)+\frac{1}{a}\left(\frac{1}{a}+\kappa^{2}\right) \gamma
$$

As $\widetilde{\nabla}_{T} T=\gamma^{\prime \prime}$ and $\kappa^{2}=c-1=\frac{4}{a}-4$, we obtain the equation of $\gamma$ (thought as a curve in $\mathbb{R}^{4}$ )

$$
a^{2} \gamma^{i v}+a(6-4 a) \gamma^{\prime \prime}+\gamma=0
$$

The general solution is

$$
\gamma(s)=\cos (A s) c_{1}+\sin (A s) c_{2}+\cos (B s) c_{3}+\sin (B s) c_{4},
$$

where $A, B$ are given by (3.2) and $\left\{c_{i}\right\}$ are constant vectors in $\mathbb{R}^{4}$.

It is easy to see that $\gamma$ must verify the following relations

$$
\begin{gathered}
\langle\gamma, \gamma\rangle=1,\left\langle\gamma^{\prime}, \gamma^{\prime}\right\rangle=\frac{1}{a},\left\langle\gamma, \gamma^{\prime}\right\rangle=0,\left\langle\gamma^{\prime}, \gamma^{\prime \prime}\right\rangle=0,\left\langle\gamma^{\prime \prime}, \gamma^{\prime \prime}\right\rangle=\frac{5-4 a}{a^{2}} \\
\left\langle\gamma, \gamma^{\prime \prime}\right\rangle=-\frac{1}{a},\left\langle\gamma^{\prime}, \gamma^{\prime \prime \prime}\right\rangle=-\frac{5-4 a}{a^{2}},\left\langle\gamma^{\prime \prime}, \gamma^{\prime \prime \prime}\right\rangle=0,\left\langle\gamma, \gamma^{\prime \prime \prime}\right\rangle=0, \\
\left\langle\gamma^{\prime \prime \prime}, \gamma^{\prime \prime \prime}\right\rangle=\frac{16 a^{2}-44 a+29}{a^{3}},
\end{gathered}
$$

Denoting $c_{i j}=\left\langle c_{i}, c_{j}\right\rangle$, in $s=0$ we have

$$
\begin{gathered}
c_{11}+2 c_{13}+c_{33}=1 \\
A^{2} c_{22}+2 A B c_{24}+B^{2} c_{44}=\frac{1}{a} \\
A c_{12}+A c_{23}+B c_{14}+B c_{34}=0 \\
A^{3} c_{12}+A B^{2} c_{23}+A^{2} B c_{14}+B^{3} c_{34}=0 \\
A^{4} c_{11}+2 A^{2} B^{2} c_{13}+B^{4} c_{33}=\frac{5-4 a}{a^{2}} \\
A^{2} c_{11}+\left(A^{2}+B^{2}\right) c_{13}+B^{2} c_{33}=\frac{1}{a} \\
A^{4} c_{22}+\left(A B^{3}+A^{3} B\right) c_{24}+B^{4} c_{44}=\frac{5-4 a}{a^{2}} \\
A^{5} c_{12}+A^{3} B^{2} c_{23}+A^{2} B^{3} c_{14}+B^{5} c_{34}=0 \\
A^{3} c_{12}+A^{3} c_{23}+B^{3} c_{14}+B^{3} c_{34}=0 \\
A^{6} c_{22}+2 A^{3} B^{3} c_{24}+B^{6} c_{44}=\frac{16 a^{2}-44 a+29}{a^{3}} .
\end{gathered}
$$


Since the determinant of the system given by (3.6), (3.7), (3.11) and (3.12) is $-A^{2} B^{2}\left(A^{2}-B^{2}\right)^{4} \neq 0$ it follows that

$$
c_{12}=c_{23}=c_{14}=c_{34}=0 .
$$

The equations (3.4), (3.8) and (3.9) give

$$
c_{11}=\frac{B}{A+B}, \quad c_{13}=0, \quad c_{33}=\frac{A}{A+B},
$$

and, from (3.5), (3.10) and (3.13) it results that

$$
c_{22}=\frac{B}{A+B}, \quad c_{24}=0, \quad c_{44}=\frac{A}{A+B} .
$$

We obtained that $\left\{c_{i}\right\}$ are orthogonal vectors in $\mathbb{R}^{4}$ with $\left\|c_{1}\right\|=\left\|c_{2}\right\|=\sqrt{\frac{B}{A+B}}$ and $\left\|c_{3}\right\|=\left\|c_{4}\right\|=\sqrt{\frac{A}{A+B}}$. Let us consider $c_{i}=\left\|c_{i}\right\| e_{i}$, where $\left\{e_{i}\right\}$ are mutually orthogonal unit constant vectors in $\mathbb{R}^{4}$.

Now, if $\gamma$ is a Legendre curve, one obtains $\langle T, \xi\rangle=0$. From the expression of $\gamma$ and since, by definition, $\xi=-J \gamma(s)$, we get $\left\langle e_{1}, J e_{2}\right\rangle=-\left\langle e_{3}, J e_{4}\right\rangle= \pm 1$ and $\left\langle e_{1}, J e_{3}\right\rangle=\left\langle e_{1}, J e_{4}\right\rangle=\left\langle e_{2}, J e_{3}\right\rangle=\left\langle e_{2}, J e_{4}\right\rangle=0$, from which arise the forms of the vectors $e_{i}$. Therefore $e_{2}=\mp J e_{1}$ and $e_{4}= \pm J e_{3}$. Finally, we obtain

$$
\begin{aligned}
\gamma(s)= & \sqrt{\frac{B}{A+B}} \cos (A s) e_{1}-\sqrt{\frac{B}{A+B}} \sin (A s) J e_{1}+ \\
& +\sqrt{\frac{A}{A+B}} \cos (B s) e_{3}+\sqrt{\frac{A}{A+B}} \sin (B s) J e_{3},
\end{aligned}
$$

and

$$
\begin{aligned}
\gamma_{1}(s)= & \sqrt{\frac{B}{A+B}} \cos (A s) e_{1}+\sqrt{\frac{B}{A+B}} \sin (A s) J e_{1}+ \\
& +\sqrt{\frac{A}{A+B}} \cos (B s) e_{3}-\sqrt{\frac{A}{A+B}} \sin (B s) J e_{3},
\end{aligned}
$$

but $\gamma$ and $\gamma_{1}$ parametrize the same curve since $\gamma_{1}(s)=\gamma(-s)$.

Remark 3.2 The geometric interpretation of equation (3.3) can be obtained as follows. Denote by $\mathbf{j}$ the canonical inclusion of $\gamma(\mathbb{R})$ in $\left(\mathbb{S}^{3}, g_{0}\right)$. Since $\langle T, T\rangle=\frac{1}{a}$, we obtain that

$$
\tau(\mathbf{j})=a \dot{\nabla}_{T} T=a \gamma^{\prime \prime}+\gamma \text { and } \tau_{2}(\mathbf{j})=a^{2} \gamma^{i v}+2 a \gamma^{\prime \prime}+(4 a-3) \gamma .
$$


Therefore $\gamma$ is a solution of (3.3) if and only if

$$
\tau_{2}(\mathbf{j})+4(1-a) \tau(\mathbf{j})=0 .
$$

We note that Riemannian immersions $\phi$ in space forms which satisfy the equation $\tau_{2}(\phi)=\lambda \tau(\phi)$, or equivalently, their mean curvature vectors are eigenvectors of the rough Laplacian, were studied (for example, see [10]).

Remark 3.3 In [3] the author studied the biharmonic curves in the Berger spheres $\mathbb{S}_{\epsilon}^{3}$. It was proved that biharmonic curves parametrized by arc length are helices and then their parametric equations were derived. We note that the Berger metric is homothetic to $g$. Therefore, by changing the parameter of the biharmonic curves of $\mathbb{S}_{\epsilon}^{3}$ we obtain the biharmonic curves of $\left(\mathbb{S}^{3}, g\right)$, and then we may select the Legendre ones. However, our method is completely different and the constant vectors are precisely determined.

\section{Proper-biharmonic Hopf cylinders}

Although proper-biharmonic curves in 3-dimensional spaces of non-constant sectional curvature were found, various attempts to find proper-biharmonic surfaces failed. Now, in the case of Hopf cylinders in $\left(\mathbb{S}^{3}, \varphi, \xi, \eta, g\right)$ we can state:

Theorem 4.1 The parametric equation of the proper-biharmonic Hopf cylinder $S_{\bar{\gamma}}$ in $\left(\mathbb{S}^{3}, \varphi, \xi, \eta, g\right)$, thought as a surface in $\left(\mathbb{R}^{4},\langle\rangle,\right)$, is

$$
\begin{aligned}
x= & x(u, v)=\sqrt{\frac{B}{A+B}} \cos \left(A u+\frac{1}{a} v\right) e_{1}-\sqrt{\frac{B}{A+B}} \sin \left(A u+\frac{1}{a} v\right) J e_{1}+ \\
& +\sqrt{\frac{A}{A+B}} \cos \left(B u-\frac{1}{a} v\right) e_{3}+\sqrt{\frac{A}{A+B}} \sin \left(B u-\frac{1}{a} v\right) J e_{3},
\end{aligned}
$$

where $\left\{e_{1}, e_{3}\right\}$ is an orthonormal system of constant vectors in the Euclidian space $\left(\mathbb{R}^{4},\langle\rangle,\right)$ with $e_{3}$ orthogonal to $J e_{1}$, and

$$
\left\{\begin{array}{l}
A=\sqrt{\frac{3-2 a-2 \sqrt{(a-1)(a-2)}}{a}} \\
B=\sqrt{\frac{3-2 a+2 \sqrt{(a-1)(a-2)}}{a}}
\end{array} .\right.
$$

Proof. We consider the Boothby-Wang fibration $\pi:\left(\mathbb{S}^{3}, g\right) \rightarrow \mathbb{C} P^{1}$, where $\mathbb{C} P^{1}$ is the complex projective space with constant sectional holomorphic curvature $\frac{4}{a}$. Let us denote by $\bar{\nabla}, \nabla, \dot{\nabla}$ and $\widetilde{\nabla}$ the Levi-Civita connections on $\mathbb{C} P^{1}$, $\left(\mathbb{S}^{3}, g\right),\left(\mathbb{S}^{3}, g_{0}\right)$ and $\left(\mathbb{R}^{4},\langle\rangle,\right)$, respectively. 
Let $S_{\bar{\gamma}}$ be a proper-biharmonic Hopf cylinder in $\left(\mathbb{S}^{3}, g\right)$, where $\bar{\gamma}: I \rightarrow \mathbb{C} P^{1}$ is the base curve parametrized by arc length. Using Theorem 2.4, $\bar{\gamma}$ has constant curvature $\bar{\kappa}= \pm \sqrt{c-1}$.

We denote $f_{1}=\bar{\gamma}^{\prime}$ and $f_{2}=\bar{J} f_{1}$. The horizontal lift $f_{1}^{H}$ of $f_{1}$ and $\xi$ form a global orthonormal frame field on $S_{\bar{\gamma}}$, and $f_{2}^{H}=\varphi f_{1}^{H}$ is normal to $S_{\bar{\gamma}}$.

In order to find the explicit parametrization of $S_{\bar{\gamma}}$ as a surface in $\mathbb{R}^{4}$, the expressions of $\widetilde{\nabla}_{f_{1}^{H}} f_{1}^{H}, \widetilde{\nabla}_{f_{1}^{H}} \xi, \widetilde{\nabla}_{f_{1}^{H}} f_{2}^{H}$ and $\widetilde{\nabla}_{\xi} f_{2}^{H}$ are needed.

First, we have that

$$
\nabla_{f_{1}^{H}} f_{1}^{H}=\left(\bar{\nabla}_{f_{1}} f_{1}\right)^{H}-g\left(f_{1}^{H}, \varphi f_{1}^{H}\right)=\bar{\kappa} f_{2}^{H} .
$$

Using again $g\left(\nabla_{X} X, Z\right)=a g_{0}\left(\dot{\nabla}_{X} X, Z\right)$, for any $Z \in C\left(T \mathbb{S}^{3}\right)$ and for any $X \in$ $C\left(T \mathbb{S}^{3}\right)$ orthogonal to $\xi$, and the properties of the Sasakian structures $(\varphi, \xi, \eta, g)$ and $\left(\varphi_{0}, \xi_{0}, \eta_{0}, g_{0}\right)$, we get

$$
\dot{\nabla}_{f_{1}^{H}} f_{1}^{H}=\bar{\kappa} f_{2}^{H}, \dot{\nabla}_{f_{1}^{H}} \xi=-\frac{1}{a} f_{2}^{H}, \dot{\nabla}_{f_{1}^{H}} f_{2}^{H}=-\bar{\kappa} f_{1}^{H}+\xi, \dot{\nabla}_{\xi} f_{2}^{H}=\frac{1}{a} f_{1}^{H} .
$$

Then

$$
\widetilde{\nabla}_{f_{1}^{H}} f_{1}^{H}=\bar{\kappa} f_{2}^{H}-\frac{1}{a} x, \widetilde{\nabla}_{f_{1}^{H}} \xi=-\frac{1}{a} f_{2}^{H}, \widetilde{\nabla}_{f_{1}^{H}} f_{2}^{H}=-\bar{\kappa} f_{1}^{H}+\xi, \widetilde{\nabla}_{\xi} f_{2}^{H}=\frac{1}{a} f_{1}^{H} .
$$

We recall that $\left[\xi, f_{1}^{H}\right]=0$, therefore we can choose a local chart $x=x(u, v)$ such that $f_{1}^{H}=x_{u}$ and $\xi=x_{v}$.

After a straightforward computation, assuming that $\bar{\kappa}=\sqrt{c-1}$, we obtain

$$
\left\{\begin{array}{l}
a^{2} x_{u u u u}+a(6-4 a) x_{u u}+x=0 \\
a x_{u u v}-\sqrt{c-1} x_{u}+x_{v}=0
\end{array} .\right.
$$

Thus

$$
\begin{aligned}
x= & x(u, v)=\cos \left(A u+\frac{1}{a} v\right) c_{1}+\sin \left(A u+\frac{1}{a} v\right) c_{2}+ \\
& +\cos \left(B u-\frac{1}{a} v\right) c_{3}+\sin \left(B u-\frac{1}{a} v\right) c_{4},
\end{aligned}
$$

where $A, B$ are given by (4.2) and $\left\{c_{i}\right\}$ are constant vectors in $\mathbb{R}^{4}$. Since

$$
\begin{gathered}
\langle x, x\rangle=1,\left\langle x, x_{u}\right\rangle=0,\left\langle x, x_{v}\right\rangle=0,\left\langle x_{u}, x_{v}\right\rangle=0 \\
\left\langle x_{u}, x_{u}\right\rangle=\frac{1}{a},\left\langle x_{v}, x_{v}\right\rangle=\frac{1}{a^{2}},\left\langle x_{v}, x_{u v}\right\rangle=0,\left\langle x_{u}, x_{u v}\right\rangle=0,
\end{gathered}
$$

one obtains, in $(u, v)=(0,0)$,

$$
c_{11}+2 c_{13}+c_{33}=1
$$




$$
\begin{gathered}
A c_{12}+B c_{14}+A c_{23}+B c_{34}=0 \\
-c_{12}+c_{14}-c_{23}+c_{34}=0 \\
-A c_{22}+(A-B) c_{24}+B c_{44}=0 \\
A^{2} c_{22}+2 A B c_{24}+B^{2} c_{44}=\frac{1}{a} \\
c_{22}-2 c_{24}+c_{44}=1 \\
-A c_{12}+A c_{14}+B c_{23}-B c_{34}=0 \\
-A^{2} c_{12}-A B c_{14}+A B c_{23}+B^{2} c_{34}=0
\end{gathered}
$$

where $c_{i j}=\left\langle c_{i}, c_{j}\right\rangle$.

From $\left\langle x_{u}, x_{u}\right\rangle=\frac{1}{a},\left\langle x_{u}, x_{v}\right\rangle=0$, one obtains, in $(u, v)=\left(0, \frac{a \pi}{2}\right)$,

$$
\begin{gathered}
A^{2} c_{11}-2 A B c_{13}+B^{2} c_{33}=\frac{1}{a} \\
-A c_{11}+(B-A) c_{13}+B c_{33}=0 .
\end{gathered}
$$

From (4.6), (4.7), (4.11) and (4.12) we have $c_{12}=c_{14}=c_{23}=c_{34}=0$. From (4.5), (4.13) and (4.14) it follows that $c_{11}=\frac{B}{A+B}, c_{13}=0, c_{33}=\frac{A}{A+B}$. Finally, from (4.8), (4.9) and (4.10) one obtains $c_{22}=\frac{B}{A+B}, c_{24}=0, c_{44}=\frac{A}{A+B}$. Thus $\left\{c_{i}\right\}$ are orthogonal vectors in $E^{4}$ with $\left\|c_{1}\right\|=\left\|c_{2}\right\|=\sqrt{\frac{B}{A+B}}$ and $\left\|c_{3}\right\|=\left\|c_{4}\right\|=\sqrt{\frac{A}{A+B}}$. Hence $c_{1}=\sqrt{\frac{B}{A+B}} e_{1}, c_{2}=\sqrt{\frac{B}{A+B}} e_{2}, c_{3}=\sqrt{\frac{A}{A+B}} e_{3}, c_{4}=\sqrt{\frac{A}{A+B}} e_{4}$, where $\left\{e_{i}\right\}$ are mutually orthogonal unit constant vectors in $\mathbb{R}^{4}$. Let us denote by $e_{i}^{k}$, $i, k=\overline{1,4}$, the components of $e_{i}$. Then the components of $x=x(u, v)$ are

$$
\begin{aligned}
x^{j}= & x^{j}(u, v)=e_{1}^{j} \cos \left(A u+\frac{1}{a} v\right)+e_{2}^{j} \sin \left(A u+\frac{1}{a} v\right)+ \\
& +e_{3}^{j} \cos \left(B u-\frac{1}{a} v\right)+e_{3}^{j} \cos \left(B u-\frac{1}{a} v\right) .
\end{aligned}
$$

Since $\xi=x_{v}(u, v), \xi=\frac{1}{a} \xi_{0}$, it follows that $e_{2}=-J e_{1}$ and $e_{4}=J e_{3}$. When $\bar{\kappa}=-\sqrt{c-1}$, following the same steps, we obtain

$$
\begin{aligned}
x_{1}(u, v)= & \sqrt{\frac{B}{A+B}} \cos \left(A u-\frac{1}{a} v\right) e_{1}+\sqrt{\frac{B}{A+B}} \sin \left(A u-\frac{1}{a} v\right) J e_{1}+ \\
& +\sqrt{\frac{A}{A+B}} \cos \left(B u+\frac{1}{a} v\right) e_{3}-\sqrt{\frac{A}{A+B}} \sin \left(B u+\frac{1}{a} v\right) J e_{3},
\end{aligned}
$$

and we see that $x(u, v)=x_{1}(-u, v)$. 
Remark 4.2 We fix now $\left\{e_{1}, e_{2}, e_{3}, e_{4}\right\}$ an orthonormal basis of $\mathbb{R}^{4}$, with $e_{2}=$ $-J e_{1}$ and $e_{4}=J e_{3}$, and consider $S_{\bar{\gamma}}$ the corresponding Hopf cylinder. We observe that the geodesics

$$
\begin{aligned}
u \rightarrow x\left(u, v_{0}\right)= & \sqrt{\frac{B}{A+B}} \cos \left(A u+\frac{1}{a} v_{0}\right) e_{1}+\sqrt{\frac{B}{A+B}} \sin \left(A u+\frac{1}{a} v_{0}\right) e_{2}+ \\
& +\sqrt{\frac{A}{A+B}} \cos \left(B u-\frac{1}{a} v_{0}\right) e_{3}+\sqrt{\frac{A}{A+B}} \sin \left(B u-\frac{1}{a} v_{0}\right) e_{4},
\end{aligned}
$$

can be written as

$$
\begin{aligned}
\gamma(s)= & \sqrt{\frac{B}{A+B}} \cos (A s) f_{1}+\sqrt{\frac{B}{A+B}} \sin (A s) f_{2}+ \\
& +\sqrt{\frac{A}{A+B}} \cos (B s) f_{3}+\sqrt{\frac{A}{A+B}} \sin (B s) f_{4},
\end{aligned}
$$

where

$$
\left\{\begin{array}{l}
f_{1}=\cos \left(\frac{1}{a} v_{0}\right) e_{1}+\sin \left(\frac{1}{a} v_{0}\right) e_{2} \\
f_{2}=-\sin \left(\frac{1}{a} v_{0}\right) e_{1}+\cos \left(\frac{1}{a} v_{0}\right) e_{2} \\
f_{3}=\cos \left(\frac{1}{a} v_{0}\right) e_{3}-\sin \left(\frac{1}{a} v_{0}\right) e_{4} \\
f_{4}=\sin \left(\frac{1}{a} v_{0}\right) e_{3}+\cos \left(\frac{1}{a} v_{0}\right) e_{4}
\end{array} .\right.
$$

Note that $\left\{f_{1}, f_{2}, f_{3}, f_{4}\right\}$ is an orthonormal basis of $\mathbb{R}^{4}$ with $f_{2}=-J f_{1}$ and $f_{4}=$ $J f_{3}$. Therefore, the only proper-biharmonic Legendre curves of $\left(\mathbb{S}^{3}, \varphi, \xi, \eta, g\right)$ are the geodesics of the biharmonic Hopf cylinders $S_{\bar{\gamma}}$ orthogonal to $\xi$.

Remark 4.3 In fact, $x$ is an isometric embedding $\mathcal{T}$ into $\left(\mathbb{S}^{3}, g\right)$, where $\mathcal{T}$ is the flat torus $\mathcal{T}=\mathbb{R}^{2} / \Lambda, \Lambda$ being the lattice generated by the vectors $w_{1}=$ $\left(\frac{2 \pi}{A+B}, \frac{2 \pi}{A(A+B)}\right)$ and $w_{2}=\left(\frac{2 \pi}{A+B},-\frac{2 \pi}{B(A+B)}\right)$.

Remark 4.4 We can obtain an alternative proof of Theorem 4.1 by using Theorem 3.1 and the flow of the Reeb vector field $\xi$.

A non-compact 3-dimensional Sasakian space form, locally isometric to $\left(\mathbb{S}^{3}, g\right)$, is provided by the Cartan-Vranceanu spaces.

In [9, the proper-biharmonic Legendre curves of the Cartan-Vranceanu 3-dimensional spaces $\left(M^{3}, d s_{l, m}^{2}\right)$ were obtained.

We recall that $M=\mathbb{R}^{3}$ if $m \geqslant 0$ and $M=\left\{(x, y, z) \in \mathbb{R}^{3}: x^{2}+y^{2}<-\frac{1}{m}\right\}$ otherwise, while

$$
d s_{l, m}^{2}=\frac{d x^{2}+d y^{2}}{\left(1+m\left(x^{2}+y^{2}\right)\right)^{2}}+\left(d z+\frac{l}{2} \frac{y d x-x d y}{\left(1+m\left(x^{2}+y^{2}\right)\right)}\right)^{2},
$$


$l, m \in \mathbb{R}$. When $l \neq 0$, the 1 -form

$$
\eta=d z+\frac{l}{2} \frac{y d x-x d y}{\left(1+m\left(x^{2}+y^{2}\right)\right)}
$$

is a contact form.

Theorem 4.5 ([9]) The parametric equations of proper-biharmonic Legendre curves of $\left(M^{3}, d s_{l, m}^{2}\right)$, with $4 m-l^{2}>0$ and $l \neq 0$, are

$$
\left\{\begin{array}{l}
x(s)=\alpha \sin \left(\beta s+c_{1}\right) \\
y(s)=-\alpha \cos \left(\beta s+c_{1}\right) \\
z(s)=\frac{l}{2} s+c_{2}
\end{array}\right.
$$

where $\beta=\frac{1+m \alpha^{2}}{\alpha}, \alpha^{2}=\frac{6 m-l^{2}+\sqrt{32 m^{2}-12 m l^{2}+l^{4}}}{2 m^{2}}$ and $c_{1}, c_{2}$ are real constants.

Moreover, if $l=2$ and $m>1$ then $\left(\mathbb{R}^{3}, d s_{2, m}^{2}\right)$ is a Sasakian space form with constant $\varphi$-sectional curvature $c=4 m-3>1$ (see [12]), and $\left(\mathbb{R}^{3}, d s_{2, m}^{2}\right)$ is locally isometric with $\left(\mathbb{S}^{3}, g\right)$.

Therefore, the cylinders along the $O z$-axis determined by (4.15), for $l=2$ and $m>1$, are the biharmonic Hopf cylinders.

\section{References}

[1] C. Baikoussis, D.E. Blair. On the geometry of 7-sphere, Results in Math., 27(1995), 5-16.

[2] C. Baikoussis, D.E. Blair, T. Koufogiorgos. Integral submanifolds of Sasakian space forms $\bar{M}^{7}$, Results in Math., 27(1995), 207-226.

[3] A. Balmus. On the biharmonic curves of the Euclidean and Berger 3dimensional spheres, Sci. Ann. USAMV Iaşi, 47(2)(2004), Proc. of the annual Symposium on "Mathematics applied in Biology \& Biophysics", May 28-29, 2004, Iaşi, 87-96.

[4] D.E. Blair. Riemannian Geometry of Contact and Symplectic Manifolds, Birkhäuser Boston, Progress in Mathematics, Volume 203(2002), pp. x, 260.

[5] R. Caddeo, S. Montaldo, C. Oniciuc. Biharmonic submanifolds of $\mathbb{S}^{3}$, Internat. J. Math., 12(2001), 867-876. 
[6] R. Caddeo, S. Montaldo, C. Oniciuc. Biharmonic submanifolds in spheres, Israel J. Math., 130(2002), 109-123.

[7] R. Caddeo, S. Montaldo, C. Oniciuc, P. Piu. The clasification of biharmonic curves of Cartan-Vranceanu 3-dimensional spaces, Modern Trends in Geometry and Topology, Deva, September 5-11, 2005, 121-131.

[8] R. Caddeo, C. Oniciuc, P. Piu. Explicit formulas for non-geodesic biharmonic curves of the Heisenberg group, Rendic. Sem. Mat. Univ. e Politecnico Torino, $62(2004), 265-278$.

[9] R. Caddeo, C. Oniciuc, P. Piu. Looking for biharmonic non-geodesic curves in SO(2)-isotropic three-dimensional manifolds, Poster, Conference "Curvature in Geometry" in honour of Prof. Lieven Vanhecke, June 11-14, 2003, Lecce.

[10] B.Y. Chen. A report on submanifolds of finite type, Soochow J. Math., 22(1996), 177-337.

[11] B.Y. Chen, S. Ishikawa. Biharmonic pseudo-Riemannian submanifolds in pseudo-Euclidean spaces, Kyushu J. Math., 52(1998), 167-185.

[12] J.T. Cho, J. Inoguchi, J.E. Lee. Biharmonic curves in 3-dimensional Sasakian space forms, Ann. Math. Pura Appl., to appear.

[13] J. Eells, J.H. Sampson. Harmonic mappings of Riemannian manifolds, Amer. J. Math., 86(1964), 109-160.

[14] J. Inoguchi. Submanifolds with harmonic mean curvature in contact 3manifolds, Colloq. Math., 100(2004), 163-179.

[15] G.Y. Jiang. 2-harmonic maps and their first and second variational formulas, Chinese Ann. Math. Ser. A7(4)(1986), 389-402.

[16] S. Montaldo, C. Oniciuc. A short survey on biharmonic maps between Riemannian manifolds, Rev. Unión Mat. Argent., to appear.

[17] K. Ogiue. On fiberings of almost contact manifolds, Kōdai Math. Sem. Rep., 17(1965), 53-62.

[18] The Bibliography of Biharmonic Maps. http://beltrami.sc.unica.it/ biharmonic/

[19] S. Tanno. The topology of contact Riemannian manifolds, Ill. J. Math., 12(1968), 700-717. 
Dorel Fetcu

Department of Mathematics Technical University of Iaşi

Bd. Carol I, no. 11

700506 Iasi

România.

e-mail: dfetcu@math.tuiasi.ro
Cezar Oniciuc

Faculty of Mathematics

"Al. I. Cuza" University of Iaşi

Bd. Carol I, no. 11

700506 Iasi

România.

e-mail: oniciucc@uaic.ro 